\title{
STAT3 promotes the proliferation and migration of hepatocellular carcinoma cells by regulating AKT2
}

\author{
YI XIE, JIANMIN LI and CHAO ZHANG \\ Department of Endoscopic Treatment and Research Center, \\ People Hospital of Henan Zhengzhou, Zhengzhou, Henan 450003, P.R. China
}

Received May 15, 2017; Accepted November 20, 2017

DOI: $10.3892 / \mathrm{ol} .2017 .7681$

\begin{abstract}
The aim of the present study was to investigate the correlation of STAT3 and AKT in HCC cells. HCC cells were transfected with si-STAT3 and si-AKT2 in vitro and the mRNA expression of STAT3 and AKT was detected by RT-PCR, and the protein expression was measured by western blot. MTT assays were used to evaluate cell proliferation, and Transwell assays were performed to detect the ability of migration and invasion. The relationship between STAT3 and AKT was analyzed by ChIP and Dual-luciferase reporter (DLR) assays. A nude mice experiment was used to verify the correlation. In the present study, we found that the expression of p-AKT2 and its downstream molecules were reduced in HCC cells transfected with si-STAT3, and the expression of p-STAT3 and its downstream molecules was decreased in HCC cells transfected with si-AKT2. Moreover, the ability of HCC cells proliferation, migration and invasion was decreased in si-STAT3 transfection group, but AKT2 reversed the role of si-STAT3 in HCC cells. The ChIP experiment found that STAT3 could bind to the AKT2 promoter in HCC cells. The DLR assay showed that the luciferase activity of AKT2 promoter was enhanced in HCC cells treated by IL-6. The nude mice experiment found that the tumor grew slowly after transfection with the STAT3-siRNA lentiviral vector, while AKT2 reversed the effect. STAT3 and AKT2 had mutual regulatory relationship, and STAT3 promoted the occurrence and development of HCC by regulating AKT2.
\end{abstract}

\section{Introduction}

Cancer has become an important factor threatening human health. Statistics show that hepatocellular carcinoma (HCC) is one of the world's high incidence of malignant tumors, and in China, it is ranked second in the highly lethal cancer (1). There

Correspondence to: $\mathrm{Dr} \mathrm{Yi}$ Xie, Department of Endoscopic Treatment and Research Center, People Hospital of Henan Zhengzhou, 7 Weiwu Road, Zhengzhou, Henan 450003, P.R. China E-mail: 174171368@qq.com

Key words: HCC, STAT3, AKT2, proliferation, migration are many factors leading to $\mathrm{HCC}$, but their nature mostly lead to the abnormal expression of the coding gene or non-coding gene (2-4), and then cause abnormal proliferation of cells and escape from the body immune system monitoring.

In recent years, STAT3 and AKT have become the two most important protooncogene. In many malignant tumors, abnormal expressions of phosphorylated STAT3 (p-STAT3) and AKT (p-AKT) are found. Signal transducer and activator of transcription (STATs) is a cytoplasmic protein family that is first found in the interferon-induced gene transcription study. It plays a key role in the signal transduction of cytokine (CK) (5). Up to now, the STATs family found in mammalian cells has STAT1, STAT2, STAT3, STAT4, STAT5 and STAT6. STAT3 was originally found as an acute phase response factors (APRF). At present, STAT3 has been found to be closely associated with tumor inflammatory response, the transformation, survival, proliferation, invasion and metastasis of tumor cells. Studies have shown that the hyperactivity of STAT3 in HCC cells can promote the proliferation, invasion and metastasis of tumor cells and inhibit the apoptosis of tumor cells, then enhance the malignant biological behavior of tumor (6). Li et al (7) found that RNA interference on STAT3 significantly inhibited cell proliferation and reduced tumor volume in mice. In addition, the activation of STAT3 can activate the expression of many downstream genes, such as Bcl-2, Bcl-xL, myeloid cell leukemia-1 (Mcl-1), X-linked inhibitor of apoptosis protein (XIAP), etc (8), and these genes have an effect on the mechanism of multiple tumors. Yang et al (9). found that evodiamine could inhibit cell proliferation and induce apoptosis by inhibiting STAT3 activity and down-regulating STAT3-mediated gene expression in HCC.

Abnormal cell signal transduction is an important factor leading to cell carcinogenesis, the signal transduction pathway will transmit the signals of abnormal growth, proliferation and differentiation to cells and lead to cancer. AKT is an important protein kinase in the signal transduction pathway, which is the downstream target protein of PI3K and the core of PI3K/AKT signal transduction pathway (10). AKT has three subtypes, which are AKT1, AKT2 and AKT3. AKT2 is one of the important subtypes of AKT, which not only has the universal characteristics of AKT, but also has its own unique biological function. AKT2 is currently considered to be a key gene of the PI3K/AKT2 signal transduction 
pathway, which mainly mediates the adhesion, movement, invasion and metastasis of PI3K-dependent cells in vivo. Researchers have transfected the full-length wild-type AKT2 cDNA into human breast and ovarian cancer cells, and the results show that transfected cells can increase tumor cell invasion and metastasis by up-regulating $\beta$-integrin (11). However, the relationship of STAT3 and AKT2 is unknown. In this paper, the correlation of STAT3 and AKT in HCC cells was investigated.

\section{Materials and methods}

Ethics. In the present study, all methods were subject to approval by the Institutional Animal Care and Use Committee of People Hospital of Henan Zhengzhou.

Cell cultures.HCC cells (SMMC7721 cells and QGY-7703 cells) were cultured in RPMI-1640 medium (FCS, ICN Biomedical Japan Co., Tokyo, Japan) containing $10 \%$ fetal bovine serum, $100 \mu \mathrm{g} / \mathrm{ml}$ streptomycin and $100 \mathrm{U} / \mathrm{ml}$ penicillin and incubated in an incubator $\left(37^{\circ} \mathrm{C}, 5 \% \mathrm{CO}_{2}\right)$. The cells used in the experiment were in the logarithmic growth phase.

Cell transfection. According to the manufacturer's instructions, si-Ctrl, si-STAT3 and si-AKT2 were transfected to the cells using Lipofectamine 2000 (Invitrogen), respectively. Before transfection, HCC cells were seeded to achieve $80-90 \%$ fusions. After $8 \mathrm{~h}$ of transfection, the supernatant was removed and washed with $1 \times$ PBS for three times, and RPMI-1640 medium was incubated with the cells for related detection. The transfection was permanent.

$R T-P C R$. The total RNA was extracted with FASTAGEN-RNAfast200 kit (Invitrogen), and the reverse transcription conditions were as follows: The reaction was carried out at $37^{\circ} \mathrm{C}$ for $15 \mathrm{~min}$ and at $85^{\circ} \mathrm{C}$ for $5 \mathrm{sec}$. The amplification reaction conditions were as follows: Pre-denaturation at $94^{\circ} \mathrm{C}$ for $5 \mathrm{~min}$, denaturation at $94^{\circ} \mathrm{C}$ for $30 \mathrm{sec}$, annealing at $50^{\circ} \mathrm{C}$ for $30 \mathrm{sec}$, extension at $30^{\circ} \mathrm{C}$ for $30 \mathrm{sec}, 30$ cycles, and then extension at $72^{\circ} \mathrm{C}$ for $5 \mathrm{~min}$. The PCR products were electrophoresed on $3 \%$ agarose gel. Bio-Rad image analysis system was used to take pictures, and Quantity One software was used to analyze the data (Bio-Rad, Berkeley, CA, USA). The relative expression quantity of the target gene was expressed by the gray scale ratio between the target band and $\beta$-actin stripe.

Western blot analysis. Cells were collected, and the total protein was extracted from the cells with RIPA lysis buffer. BCA protein quantitative kits (Invitrogen) were used to measure protein sample concentration. $50 \mu \mathrm{g}$ total protein were separated by SDS-PAGE and transferred to PVDF membranes, and then incubated with STAT3, p-STAT3, AKT2, p-AKT2, mTOR, p-mTOR, Cyclin D1, Bcl-xl and $\beta$-actin antibody (Guangzhou RiboBio Co., Ltd., Guangzhou, China) overnight at $4^{\circ} \mathrm{C}$ after the addition of $50 \mathrm{mg} / 1$ skimmed milk powder for $1.5 \mathrm{~h}$. Finally, the membranes were washed for 3 times and incubated with horseradish peroxidase (HRP)-conjugated secondary antibody $(1: 1,000)$ at room temperature for $2 \mathrm{~h}$, and developed by ECI.
MTT assay. SMMC7721 cells and QGY-7703 cells were divided into four groups (si-Ctrl group, si-STAT3 group, si-STAT3+pcDNA group, si-STAT3+pcDNA-AKT2 group) and inoculated into 96 -well plates $\left(2 \times 10^{5}\right.$ cells/well). On the second day of inoculation, 6 wells were set in each group, and $20 \mu \mathrm{l}$ of MTT solution $(5 \mathrm{mg} / \mathrm{ml})$ were added to each well. After incubation at $37^{\circ} \mathrm{C}$ for $4 \mathrm{~h}$, the supernatant was removed and $150 \mu \mathrm{l}$ of dimethyl sulfoxide (DMSO) was added to each well, and then the absorbance of each well was measured by a microplate reader (wavelength $492 \mathrm{~nm}$ ) within $1 \mathrm{~h}$ after mixing.

Migration and invasion assays. Transwell chambers $(8 \mathrm{~mm}$ pore size; Corning Incorporated, Corning, NY, USA) were used to determine the ability of migration and invasion in HCC cells. The experimental grouping method was the same as section 2.5. For migration assay, cells were digested with trypsin and made into $2 \times 10^{5}$ cells $/ \mathrm{ml}$ of cell suspension. $200 \mu \mathrm{l}$ of cell suspension were added to each well of the upper chamber of the Transwell, and $500 \mu \mathrm{l}$ of 1640 medium containing serum was added to the lower chamber. After $48 \mathrm{~h}$ of incubation, the cells on the upper surface were removed by a cotton swab, and the cells on the lower surface of the membrane were fixed with $90 \%$ alcohol for $30 \mathrm{~min}$. Then, the cells were stained with crystal violet for $20 \mathrm{~min}$ and rinsed with water. Cells were counted under a microscope in 5 random fields and the results were calculated as a percentage.

The invasion assay, $50 \mu \mathrm{l}$ Matrigel was added to the upper surface of the polycarbonate film of the transwell chamber to solidify the gel. $500 \mu \mathrm{l}$ of 1640 medium containing serum was added to the lower chamber. After $48 \mathrm{~h}$ of incubation, the cells were treated as described above.

Chromatin immunoprecipitation assay. According to the manufacturer's instructions, the Chromatin Immunoprecipitation (ChIP) assay was carried out with ChIP assay kits (EMD Millipore, Billerica, MA, USA). Briefly, the IL-6 stimulated (400 U/ml) and unstimulated SMMC7721 cells were fixed with $1 \%$ formaldehyde at room temperature for $10 \mathrm{~min}$ and neutralized with glycine for $5 \mathrm{~min}$, followed by removing the supernatant and washing with 1xPBS for twice. Then, the cells were lysed and collected in $1.5 \mathrm{ml}$ EP tube (RNase-free). The RNA fragment was disrupted by ultrasound for $5 \mathrm{~min}$, and supernatant was collected after centrifugation. Then, si-STAT3 and IgG antibody was added into supernatant and incubated overnight at $4^{\circ} \mathrm{C}$. After that, protein $\mathrm{A} / \mathrm{G}$ was added to complexes and incubated at $4^{\circ} \mathrm{C}$ for $2 \mathrm{~h}$. After washing the pellets, elution buffer was added and the samples were heated at $65^{\circ} \mathrm{C}$ for $1 \mathrm{~h}$ to remove cross-linking. Trizol method was uesd to extract RNA and PCR products were electrophoresed on $3 \%$ agarose gel, and the following steps were described as above.

Dual-Luciferase reporter gene assay. SMMC7721 cells were inoculated into 24 -well plates $\left(1 \times 10^{5}\right.$ cells/well) and tested with Dual-Luciferase Reporter Assay kit (DLR; Promega Corp., Madison, WI, USA) according to the corresponding kit instructions. Briefly, $100 \mu \mathrm{l}$ cell lysate was added to each well and shaken slowly at room temperature for $15 \mathrm{~min}$, and then adding $100 \mu \mathrm{l}$ luciferase assay reagent II (LARII) to cell lysate $(20 \mu \mathrm{l})$. Firefly luciferase activity was detected 
by fluorescence luminescence detector. Then, adding $100 \mu \mathrm{l}$ renilla luciferase detection reagent to determine renilla luciferase activity. The ratio of Firefly and TK Renilla Luciferase activity was used as the reporter gene activity value. TK Renilla fluorescence value was used as an internal reference.

Animal experiment. Forty nude mice were randomly divided into si-Ctrl group (control group), si-STAT3 group, si-STAT3+pcDNA group and si-STAT3+pcDNA-AKT2 group, and each group had 10 mice. In si-STAT3 group, $0.1 \mathrm{ml}$ (containing 1.0x10 $10^{7}$ cells) SMMC-7721/si-STAT3 cell suspension (SMMC-7721 cells transfected with STAT3-siRNA lentiviral vector) or QGY-7703/si-STAT3 cell suspension (QGY-7703 cells transfected with STAT3-siRNA lentiviral vector) was subcutaneously inoculated to the left armpit of nude mouse. In si-STAT3+pcDNA-AKT2 group, $0.1 \mathrm{ml} \mathrm{HCC} \mathrm{cell}$ suspension (SMMC-7721 cells transfected with si-STAT3 lentiviral vector and pcDNA-AKT2 or QGY-7703 cells transfected with si-STAT3 lentiviral vector and pcDNA-AKT2) was subcutaneously inoculated in nude mouse. The control group was inoculated with SMMC7721 cell suspension or QGY-7703 cell suspension. The long diameter (a) and short diameter (b) of the tumor was measured with a vernier caliper every week, and the tumor volume $(\mathrm{V})$ was calculated with the formula $\mathrm{V}=0.5 \mathrm{xaxb}^{2}$.

Statistical analysis. SPSS 19.0 statistical software was used to analyze the data. Student's t-test or one-way analysis of variance (ANOVA) was used to compare quantitative variables. All data were expressed as means \pm standard deviation (SD). $\mathrm{P}<0.05$ was considered statistically significant.

\section{Results}

Effect of si-STAT3 on the expression of STAT3 and AKT2 in HCC cells. In the present study, si-STAT3 or si-Control was transfected to SMMC7721 cells and QGY7703 cells. The transfection was permanent. As indicated in Fig. 1, after $48 \mathrm{~h}$ of transfection, STAT3 and AKT2 expression was detected by RT-PCR and western blot respectively. Compared with the si-Control, in SMMC7721 cells transfected with si-STAT3, the mRNA level of STAT3 and AKT2 was significantly reduced (Fig. 1A), and the protein levels of p-STAT3 ${ }^{\text {(Tyr705) }}$, p-AKT2 ${ }^{\text {(Ser473) }}$ and $\mathrm{p}-\mathrm{mTOR}^{(\mathrm{Ser} 244)}$ (downstream molecule of AKT2) were markedly decreased (Fig. 1C). These results indicated that transfection with si-STAT3 was effective in SMMC7721 cells. Similar results were observed in QGY7703 cells (Fig. 1B and D).

Effect of si-AKT2 on the expression of AKT2 and STAT3 in HCC cells. In the present study, si-AKT2 or si-Control was transfected to SMMC7721 cells and QGY7703 cells. The transfection was permanent. As indicated in Fig. 2, after $48 \mathrm{~h}$ of transfection, AKT2 and STAT3 expression was detected by RT-PCR and western blot respectively. Compared with the si-Control, in SMMC7721 cells transfected with si-AKT2, the mRNA level of AKT2 and STAT3 was significantly decreased (Fig. 2A), and the protein levels of p-AKT2, p-STAT3 and its downstream molecules (Cyclin D1 and Bcl-xl) were obviously reduced (Fig. 2C). These results suggested that transfection with si-AKT2 was effective in SMMC7721 cells. Similar results were obtained in QGY7703 cells (Fig. 2B and D).
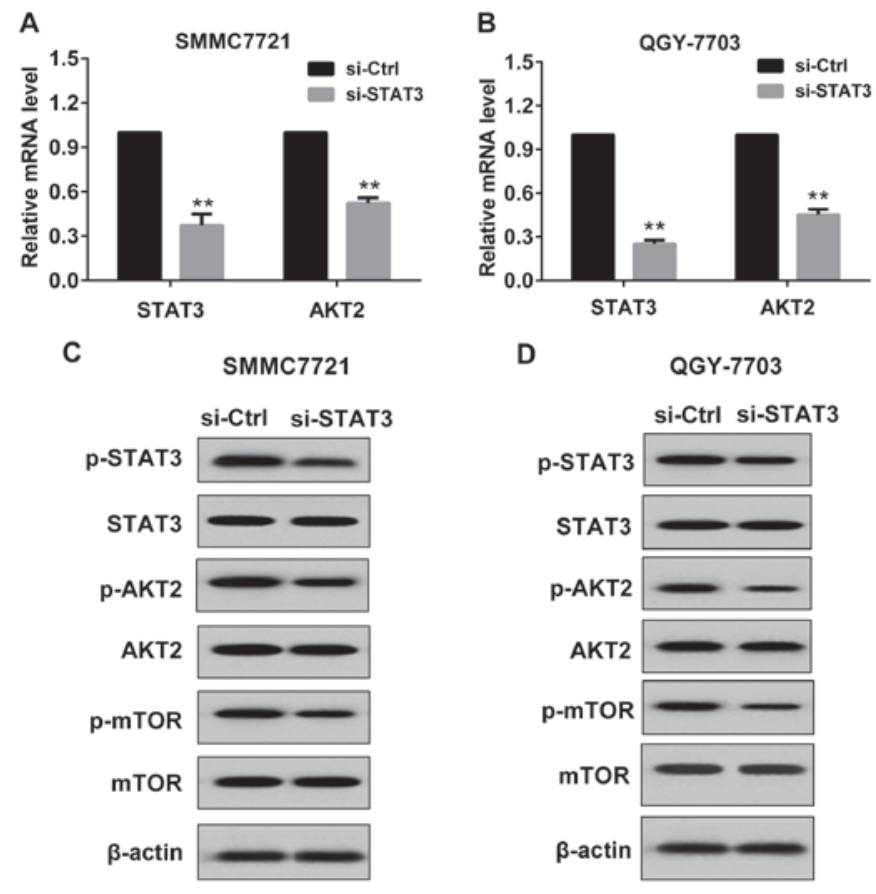

Figure 1. Effect of si-STAT3 on the expression of STAT3 and AKT2 in HCC cells. In SMMC7721 cells and QGY-7703 cells transfected with si-STAT3, (A and B) the mRNA level of STAT3 and AKT2 was significantly reduced, (C and D) the protein levels of p-STAT3, p-AKT2 and p-mTOR (downstream molecule of p-AKT2) were significantly decreased. ${ }^{* *} \mathrm{P}<0.05$, vs. si-Ctrl.
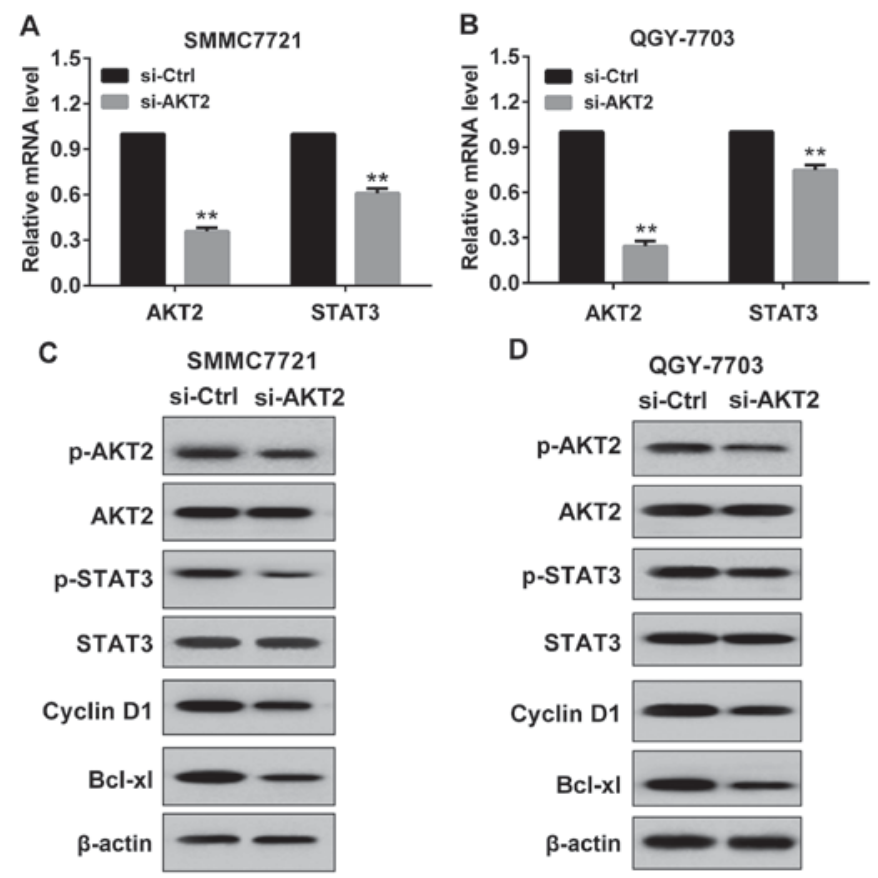

Figure 2. Effect of si-AKT2 on the expression of AKT2 and STAT3 in HCC cells. (A and B) In SMMC7721 cells and QGY-7703 cells transfected with si-AKT2, the mRNA level of STAT3 and AKT2 was significantly reduced, (C and D) the protein levels of p-AKT2, p-STAT3 and its downstream molecule (Cyclin D1 and Bcl-xl) were significantly decreased. ${ }^{* *} \mathrm{P}<0.05$, vs. si-Ctrl.

Effect of si-STAT3 and AKT2 on the proliferation, migration and invasion of HCC cells. HCC cells were divided into si-Control group, si-STAT3 group, si-STAT3+pcDNA group and si-STAT3+pcDNA-AKT2 group. MTT analysis (Fig. 3) 
A
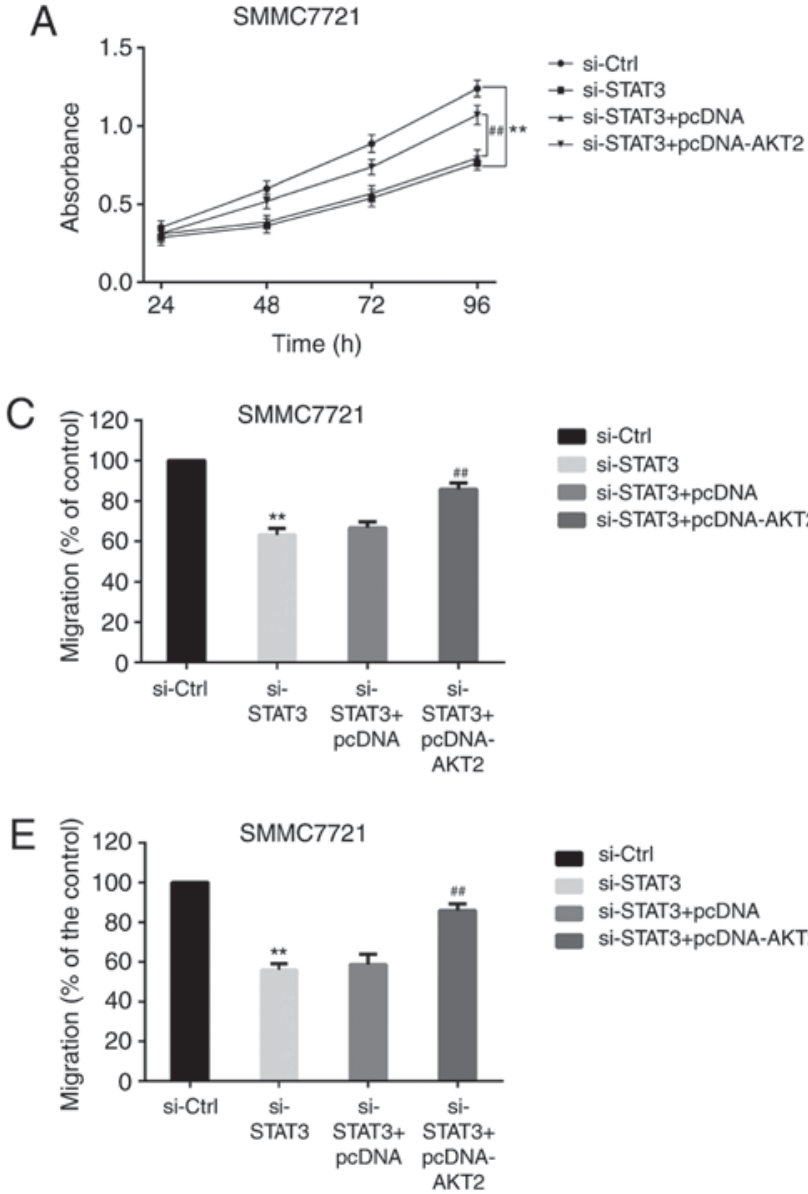

B
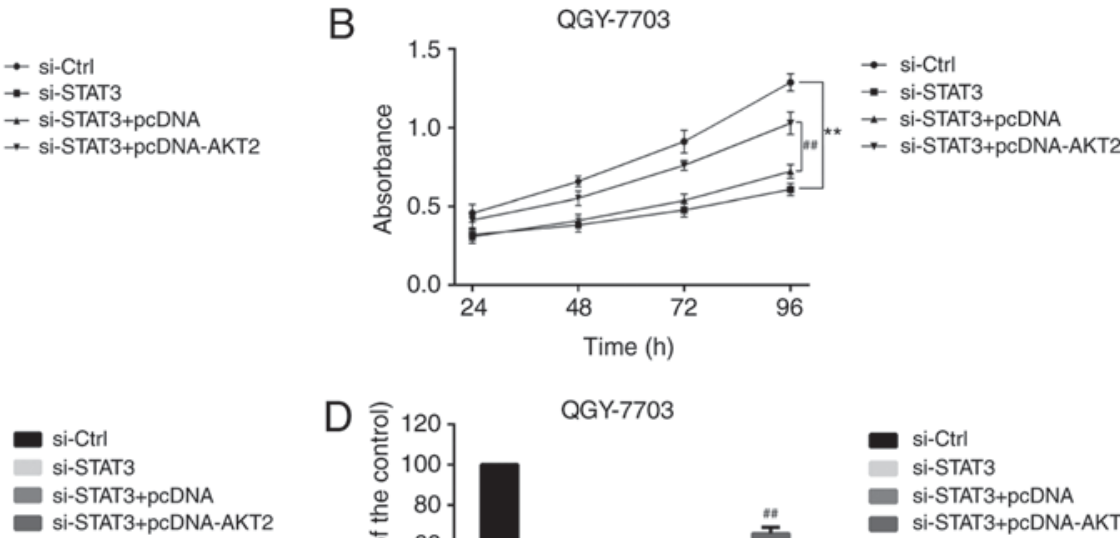

D
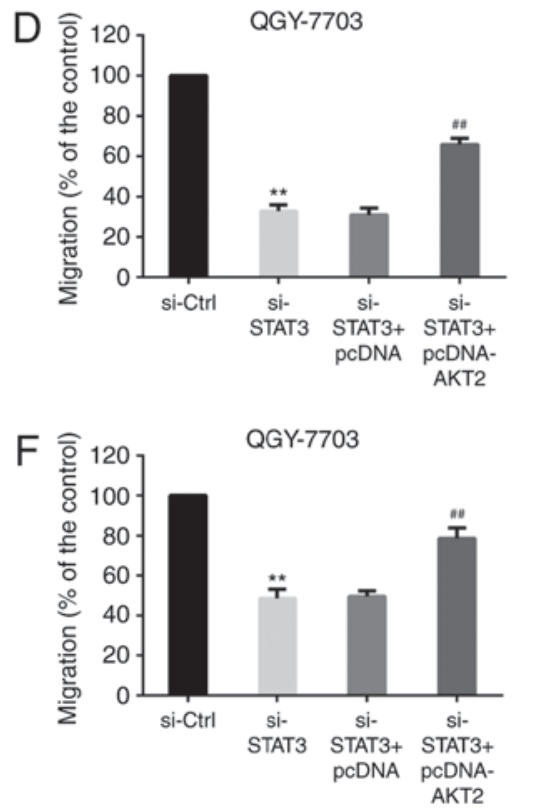

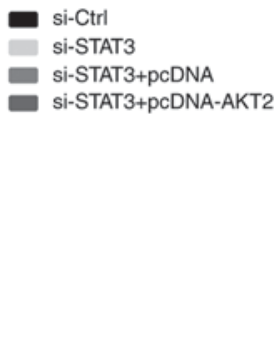

si-Ctrl si-STAT3 si-STAT3+PcDNA si-STAT3+PCDNA-AKT2

Figure 3. Effect of si-STAT3 and AKT2 on the proliferation, migration and invasion of HCC cells. (A and B) The absorbance of SMMC-7721 cells and QGY-7703 cells was lower in si-STAT3 group than in si-Control group. And compared with si-STAT3+pcDNA group, in si-STAT3+pcDNA-AKT2 group, the absorbance of HCC cells was increased. (C and D) The migration of SMMC-7721 cells and QGY-7703 cells was reduced in si-STAT3 group when compared with si-Control group. In contrast, the migration of HCC cells was increased in si-STAT3+pcDNA-AKT2 group. (E and F) Compared with si-Control group, the invasion of SMMC-7721 cells and QGY-7703 cells was decreased in si-STAT3 group. And compared with si-STAT3+pcDNA group, the invasion of HCC cells was increased in si-STAT3+pcDNA-AKT2 group. ${ }^{* *} \mathrm{P}<0.05$, vs. si-Ctrl. ${ }^{\# \#} \mathrm{P}<0.05$, vs. si-STAT3+pcDNA.

showed that the absorbance of SMMC-7721 cells (Fig. 3A) and QGY-7703 cells (Fig. 3B) was lower in si-STAT3 group than in si-Control group. And compared with si-STAT3+pcDNA group, the absorbance of HCC cells (Fig. 3A and B) was increased in si-STAT3+pcDNA-AKT2 group. The results illustrated that si-STAT3 could reduce the proliferation of HCC cells, and AKT2 could change the phenomenon.

Transwell assays demonstrated that the migration of SMMC-7721 cells (Fig. 3C) and QGY-7703 cells (Fig. 3D) was reduced in si-STAT3 group when compared with si-Control group. In contrast, the migration of HCC cells (Fig. 3C and D) was increased in si-STAT3+pcDNA-AKT2 group when compared with si-STAT3+pcDNA group. Similarly, compared with si-Control group, the invasion of SMMC-7721 cells (Fig. 3E) and QGY-7703 cells (Fig. 3F) was decreased in si-STAT3 group. And compared with si-STAT3+pcDNA group, the invasion of HCC cells (Fig. 3E and F) was increased in si-STAT3+pcDNA-AKT2 group. These data indicated that STAT3 regulated the proliferation, migration and invasion of $\mathrm{HCC}$ by AKT2.

Effect of STAT3 on AKT2 expression. As demonstrated in Fig. 4, the ChIP assay showed that STAT3 could bind to AKT2 promoter in SMMC-7721 cells, while no binding was detected in cells transfected with si-STAT3 (Fig. 4A). Compared with the control group (IgG stimulated SMMC7721 cells), the mRNA levels of STAT3 and AKT2 in IL-6 (a known inducer of STAT3) stimulated SMMC7721 cells were increased (Fig. 4B), and the protein levels of p-STAT3 and p-AKT2 were also enhanced (Fig. 4B). Moreover, ChIP assay showed STAT3 could better combine with AKT2 promoter after IL-6 stimulation (Fig. 4C). DLR assay showed that the fluorescence activity of the luciferase reporter vector containing the AKT2 promoter sequence in SMMC7721 cells transfected with si-STAT3 was significantly lower than that of the no-load without the AKT2 promoter sequence (Fig. 4D). However, in SMMC7721 cells treated with IL-6, the fluorescence activity of the luciferase reporter vector containing the AKT2 promoter sequence was significantly higher than that of the no-load without the AKT2 promoter sequence (Fig. 4D).

si-STAT3 inhibits tumor growth of hepatoma xenografts in nude mice. As indicated in Fig. 5, from the second week, nude mice were subcutaneously inoculated with SMMC-7721 cells and QGY7703 cells, the volume of tumor in si-STAT3 group was significantly smaller than that in si-Control 


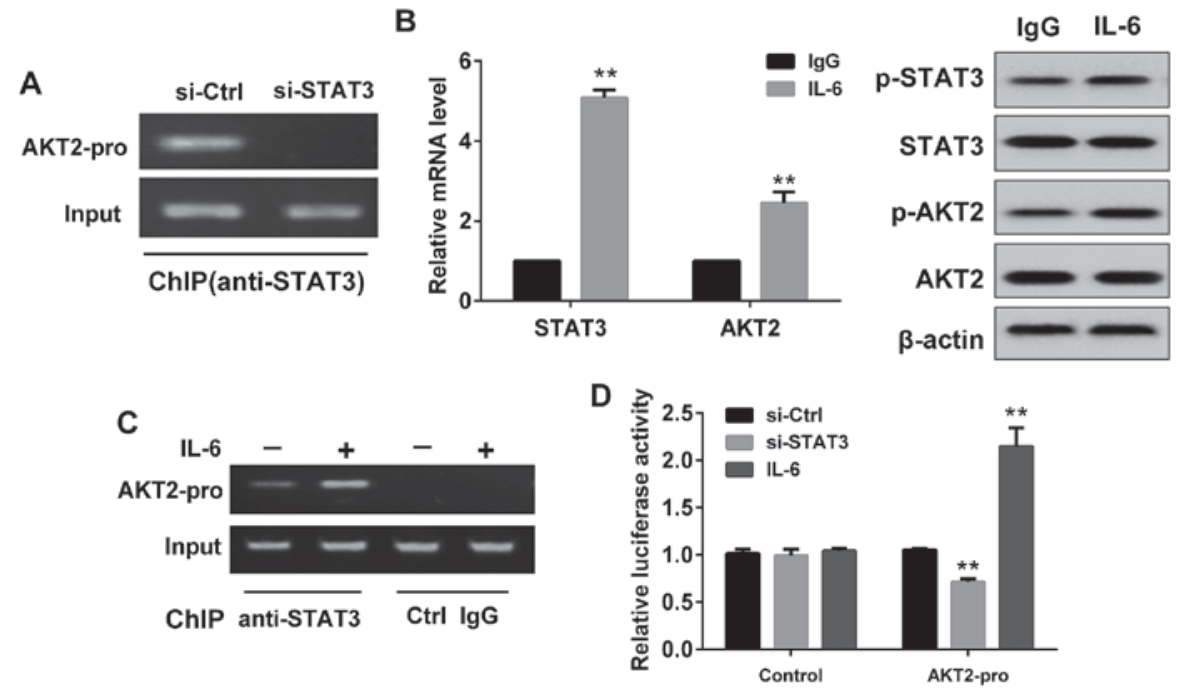

Figure 4. STAT3 directly regulated AKT2 expression in HCC cells. (A) ChIP assay showed that STAT3 could bind to AKT2 promoter (AKT2-pro) in SMMC-7721 cells, while no binding was detected in cells transfected with si-STAT3. (B) The mRNA expression of STAT3 and AKT2 in IL-6-stimulated (400 U/ml) SMMC-7721 cells was detected by RT-PCR (upper), and a western blotting assay was used to examine the level of phosphorylated STAT3 (p-STAT3) and phosphorylated AKT2 (p-AKT2) in SMMC-7721 cells (upper). (C) A ChIP-PCR assay was performed using an anti-STAT3 antibody or rabbit IgG as a control. The results showed that STAT3 could better combine with AKT2 promoter after IL-6 stimulation. (D) DLR assay showed that the luciferase activity of AKT2-pro in SMMC7721 cells transfected with si-STAT3 was significantly reduced, and in SMMC7721 cells treated with IL-6, the luciferase activity was significantly increased. ${ }^{* *} \mathrm{P}<0.05$, vs. si-Ctrl or IgG.
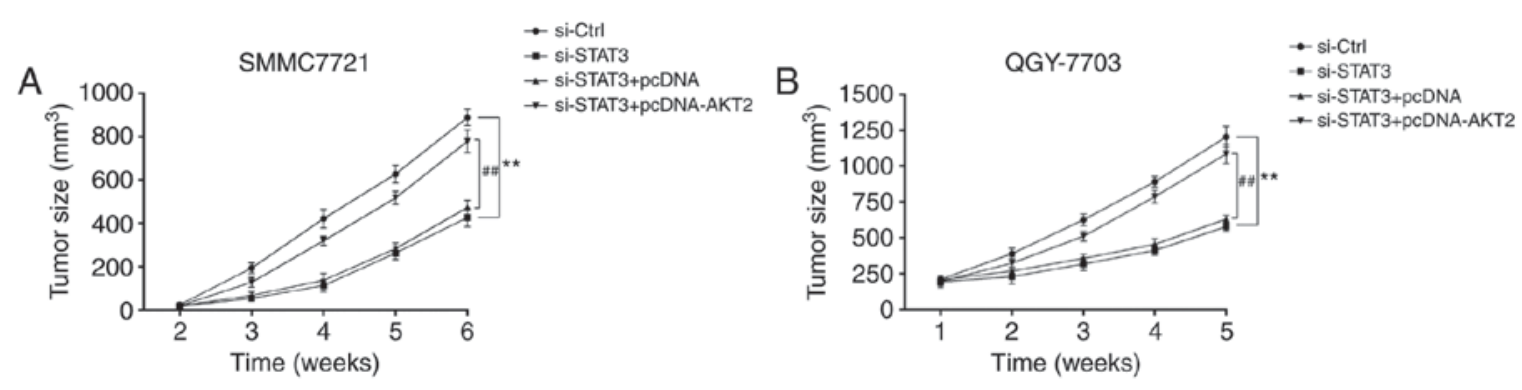

Figure 5. si-STAT3 inhibits tumor growth of hepatoma xenografts in nude mice. Nude mice were subcutaneously inoculated with SMMC-7721 cells and QGY7703 cells. (A and B) The tumor size in si-STAT3 group was significantly smaller than that in si-Control group. However, the tumor size in si-STAT3+pcDNA-AKT2 group was markedly higher than that in si-STAT3+pcDNA group. ${ }^{* *} \mathrm{P}<0.05$, vs. si-Ctrl. ${ }^{\# \prime} \mathrm{P}<0.05$, vs. si-STAT3+pcDNA.

group (Fig. 5A and B). However, the tumor volume in si-STAT3+pcDNA-AKT2 group was markedly higher than that in si-STAT3+pcDNA group (Fig. 5A and B).

\section{Discussion}

HCC is the fourth most common malignant tumor in the world. Although surgical treatment and adjuvant chemotherapy are continually improving, the survival rate of patients with HCC is always very low. At present, due to the development of chemotherapy, molecular targeted therapy and gene therapy, these technologies are expected to bring new hope to patients with HCC.

Studies have shown that abnormal activation of STAT3 is often associated with the occurrence of tumors (12), which can inhibit tumor cell apoptosis and enhance the tolerance of tumor cells to chemotherapeutic drugs (13). At the same time, p-STAT3 cannot only activate the expression of genes related to proliferation, apoptosis and invasion, but also inhibit the expression of genes associated with anti-tumor responses (14-17). It had been reported that RNA interference
STAT3 could significantly inhibit cell proliferation and reduce the tumor volume of tumor-bearing mice (7). In addition, Li et al (18) found that blocking the expression of STAT3 by interfering w (16) ith RNA interference (RNAi) significantly inhibited the expression of Cyclin D1 and Bcl-2 in cancer cells and promoted the apoptosis of tumor cells. Kunigal et al (19) reported that blocking the expression of STAT3 by RNAi could up-regulate the expression of Fas protein, down-regulate Bcl-xL protein, and promote the apoptosis of tumor cells. In this study, we had similar findings. We discovered that silencing STAT3 by RNAi could reduce mRNA level of AKT2 in HCC cells, down-regulate the expression of p-AKT2 and its downstream molecules, and inhibit the proliferation, migration and invasion of HCC cells. Moreover, we also found that silencing AKT2 by RNAi could attenuate the expression of p-STAT3 and its downstream molecules in HCC cells. These results indicated that STAT3 and AKT2 could interact with each other. However, the mechanism between them would need further study.

AKT2 is defined as an oncogene $(20,21)$, which is closely related to the occurrence and development of HCC (22). In the present study, we found AKT2 could reverse the inhibitory 
effect of si-STAT3 in HCC cells. Therefore, we speculated that STAT3 might promote the proliferation, migration and invasion of HCC cells by regulating AKT2. Further research confirmed this hypothesis. ChIP experiment demonstrated that STAT3 in HCC cells could bind to AKT2 promoter, and the combination was more closely after IL-6 (a known inducer of STAT3) stimulation, while no binding was found in HCC cells transfected with STAT3. Dual-Luciferase reporter gene assay indicated that STAT3 could promote transcription of AKT2 by binding to the AKT2 promoter, and then increase AKT2 expression. Thus, the research demonstrated for the first time that STAT3 regulated AKT2 expression in HCC cells by a direct mechanism. Furthermore, nude mice experiment also confirmed this finding.

In this study, we chose SMMC7721 and QGY-7703 cell lines as research objects because the two hepatoma cell lines could grow more rapidly and stably, and have a high the tumor formation rate in immunodeficient mice. However, the two hepatoma cell lines exhibited some differences in the intensity of their responses to the treatments. We believed that it was related to the origin of the two hepatoma cell lines. SMMC7721 cell line comes from a male liver tissue, and QGY-7703 cell line comes from a female liver tissue. Additionally, it inevitably has some improper operation during the experimental, which is also the cause of the difference.

In conclusion, our results revealed that STAT3 might promote the occurrence and development of hepatocellular carcinoma by regulating AKT2. And the result shed a new light on therapeutic target for HCC.

\section{Acknowledgements}

This research was supported by Basic and frontier technology research project of Henan Provincial Department of science and technology in 2014 (NO.142300410073).

\section{References}

1. Jemal A, Bray F, Center MM, Ferlay J, Ward E and Forman D: Global cancer statistics. CA Cancer J Clin 61: 69-90, 2011.

2. Huang J, Wang Y, Guo Y and Sun S: Down-regulated microRNA-152 induces aberrant DNA methylation in hepatitis $B$ virus-related hepatocellular carcinoma by targeting DNA methyltransferase 1. Hepatology 52: 60-70, 2010.

3. Feitelson MA, Sun B, Satiroglu Tufan NL, Liu J, Pan J and Lian Z: Genetic mechanisms of hepatocarcinogenesis. Oncogene 21: 2593-2604, 2002.

4. Hou J, Zhou Y, Zheng Y, Fan J, Zhou W, Ng IO, Sun H, Qin L, Qiu S, Lee JM, et al: Hepatic RIG-I predicts survival and interferon- $\alpha$ therapeutic response in hepatocellular carcinoma. Cancer Cell 25: 49-63, 2014.

5. Darnell JE Jr: STATs and gene regulation. Science 277: 1630-1635, 1997.
6. Yuen JW, Poon LS, Chan AS, Yu FW, Lo RK and Wong YH: Activation of STAT3 by specific galpha subunits and multiple gbetagamma dimers. Int J Biochem Cell Biol 42: 1052-1059, 2010.

7. Li WC, Ye SL, Sun RX, Liu YK, Tang ZY, Kim Y, Karras JG and Zhang $\mathrm{H}$ : Inhibition of growth and metastasis of human hepatocellular carcinoma by antisense oligonucleotide targeting signal transducer and activator of transcription 3. Clin Cancer Res 12: 7140-7148, 2006.

8. Levy DE and Darnell JE Jr: Stats: Transcriptional control and biological impact. Nat Rev Mol Cell Biol 3: 651-662, 2002.

9. Yang J, Cai X, Lu W, Hu C, Xu X, Yu Q and Cao P: Evodiamine inhibits STAT3 signaling by inducing phosphatase shatterproof 1 in hepatocellular carcinoma cells. Cancer Lett 328: 243-251, 2013.

10. Pham NA, Tsao MS, Cao P and Hedley DW: Dissociation of gemcitabine sensitivity and protein kinase B signaling in pancreatic ductal adenocarcinoma models. Pancreas 35: e16-e26, 2007.

11. Arboleda MJ, Lyons JF, Kabbinavar FF, Bray MR, Snow BE, Ayala R, Danino M, Karlan BY and Slamon DJ: Overexpression of AKT2/protein kinase Bbeta leads to up-regulation of beta1 integrins, increased invasion and metastasis of human breast and ovarian cancer cells. Cancer Res 63: 196-206, 2003.

12. Lee H, Herrmann A, Deng JH, Kujawski M, Niu G, Li Z, Forman S, Jove R, Pardoll DM and Yu H: Persistently activated Stat 3 maintains constitutive NF- $\kappa \mathrm{B}$ activity in tumors. Cancer Cell 15: 283-293, 2009.

13. Sansone P and Bromberg J: Targeting the interleukin-6/Jak/stat pathway in human malignancies. J Clin Oncol 30: 1005-1014, 2012.

14. Fukuda A, Wang SC, Morris JP IV, Folias AE, Liou A, Kim GE, Akira S, Boucher KM, Firpo MA, Mulvihill SJ and Hebrok M: Stat 3 and MMP7 contribute to pancreatic ductal adenocarcinoma initiation and progression. Cancer Cell 19: 441-455, 2011.

15. Kortylewski M, Xin H, Kujawski M, Lee H, Liu Y, Harris T, Drake C, Pardoll D and Yu H: Regulation of the IL-23 and IL-12 balance by Stat 3 signaling in the tumor microenvironment. Cancer Cell 15: 114-123, 2009.

16. Lesina M, Kurkowski MU, Ludes K, Rose-John S, Treiber M, Klöppel G, Yoshimura A, Reindl W, Sipos B, Akira S, et al: Stat $3 /$ Socs 3 activation by IL- 6 transsignaling promotes progression of pancreatic intraepithelial neoplasia and development of pancreatic cancer. Cancer Cell 19: 456-469, 2011.

17. Li N, Grivennikov SI and Karin M: The unholy trinity: Inflammation, cytokines and STAT3 shape the cancer microenvironment. Cancer Cell 19: 429-431, 2011.

18. Li GH, Wei H, Lv SQ, Ji H and Wang DL: Knockdown of STAT3 expression by RNAi suppresses growth and induces apoptosis and differentiation in glioblastoma stem cells. Int J Oncol 37: 103-110, 2010.

19. Kunigal S, Lakka SS, Sodadasu PK, Estes N and Rao JS: Stat3-siRNA induces fas-mediated apoptosis in vitro and in vivo in breast cancer. Int J Oncol 34: 1209-1220, 2009.

20. Rössig L, Jadidi AS, Urbich C, Badorff C, Zeiher AM and Dimmeler S: Akt-dependent phosphorylation of p21Cip1 regulates PCNA binding and proliferation of endothelial cells. Mol Cell Biol 21: 5644-5657, 2001.

21. Shah A, Swain WA, Richardson D, Edwards J, Stewart DJ, Richardson CM, Swinson DE, Patel D, Jones JL and O'Byrne KJ: Phospho-akt expression is associated with a favorable outcome in non-small cell lung cancer. Clin Cancer Res 11: 2930-2936, 2005.

22. Barderas R,Mendes M, Torres S, Bartolomé RA,López-Lucendo M, Villar-Vázquez R, Peláez-García A, Fuente E, Bonilla F and Casal JI: In-depth characterization of the secretome of colorectal cancer metastatic cells identifies key proteins in cell adhesion, migration and invasion. Mol Cell Proteomics 12: 1602-1620, 2013. 